\title{
Setting Safety Standards by Designing a Low-budget and Compatible Patient Identification System based on Passive RFID Technology
}

\author{
Dr Thuemmler C (1), Prof Buchanan W (2) and Kumar V (2) \\ (1) University Hospital, Birmingham, UK \\ (2) Centre for Distributed Computing and Security, Napier University, UK
}

\section{Abstract}

This paper outlines a large-scale audit for the enhancement of quality of care and staff and patient safety using passive RFID (Radio Frequency ID) wrist bands, which link to a patient's database, in order to reduce errors in patient care. It has been developed with a collaboration between the University Hospital, Birmingham and UK, Napier University, UK. The key features of the work is the usage of passive RFID tags as an integral part of a low budget out-of-the box strategic patient information system which should be compatible with most hospital IT systems.

\section{INTRODUCTION}

$\mathrm{R}$ esearch and anecdotal evidence has shown that patients often do not have wristbands or carry misleading or inappropriate identification. These errors can thus increase the risk of patients being incorrectly identified, and given the wrong care [1-6]. To highlight the problem, between November 2003 and July 2005, the National Patient Safety Agency (NPSA) in the UK received 236 reports of patient safety incidents and near misses relating to missing wristbands or wristbands with incorrect information [20].

Normally conventional wristbands consist of plastic strips with patient identification labels from paper attached to the surface. In some cases, patient names are simply written on the tag as ones which are phonetically understood by nursing staff. Thus, simple autographic mistakes, or the lack of secondary identification, like the date-of-birth, quite frequently make the whole process of patient identification prone to error by mixed identity. Another source of failure is the vulnerability of the paper stickers to chemical agents like soap or disinfectants. Stickers often become unreadable, or they dissolve causing extreme difficulties with identification. Along with this, there is currently no standard color used for wristbands for allergy and infection details. It has been observed that medical professionals can go from one hospital to another, and the other hospital uses different colors for these details.

All this needs to be understood in context with a growing number of patients who are not able to confirm their personal data, secondary to their conditions such as acute confusion, dementia, depression, intoxication, stroke, and so on. The rapidly developing area of RFID technology offers an interesting and novel approach towards intelligent and highly reliable patient identification systems. Thus, labels and stickers could be abandoned, as wireless sensors linked to memory can be embedded in wristbands and can be read either by handheld devices, or by antennas positioned in the hospital. With the enhancements in the authentication and privacy in wireless technology, such as the developments in WPA, allow a secure environment for collection, preservation, analysis and report of the data.

\section{BACKGROUND}

Bar codes have been used extensively in commerce, especially in identifying products to give a Unique Product Code (UPC). In fact, the first one was on a pack of Wrigley's chewing gum on June 26, 1974. The example in Figure 1, from a book, shows that the ISBN code is mapped to the bar code identifier. Unfortunately, bar codes suffer from several weaknesses, including:

- Bar code readers normally require a line-of-sight transmission.

- $\quad$ RFID can be read from a bigger distance.

- They must be viewable on the surface of an object.

- Bar codes only contain manufacturer and product.

The enhancement to bar codes is to use a wireless transmission mechanism with local memory to store information. This type of technology has many applications, including pet tracking, automated checkouts, food safety, remote telemetry, ID cards, automated tolls, and so on (Figure 2). This type of technology, typically name RFID, has several advantages, including:

- RFID do not require a line-of-sight transmission.

- RFID can be embedded into a device, and thus more robust.

- RFID can contain other information, such as a unique ID, manufacturing factory.

The radio frequencies that these tags use have been fitted into three main gaps in the RF (Radio Frequency) spectrum (Figure 3), and most common tags use the $13.56 \mathrm{MHz}$ frequency, which is a globally defined standard. This allows the tags to have a reasonable range, while still having a relevantly small antenna. The usage of high-frequency tags gives the opportunity for extremely small tags, but their range tends to be less wide than lower frequency tags.

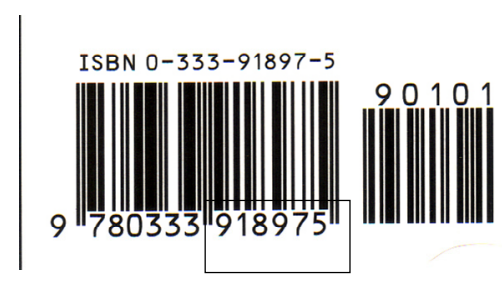

Figure 1: Example Bar code 


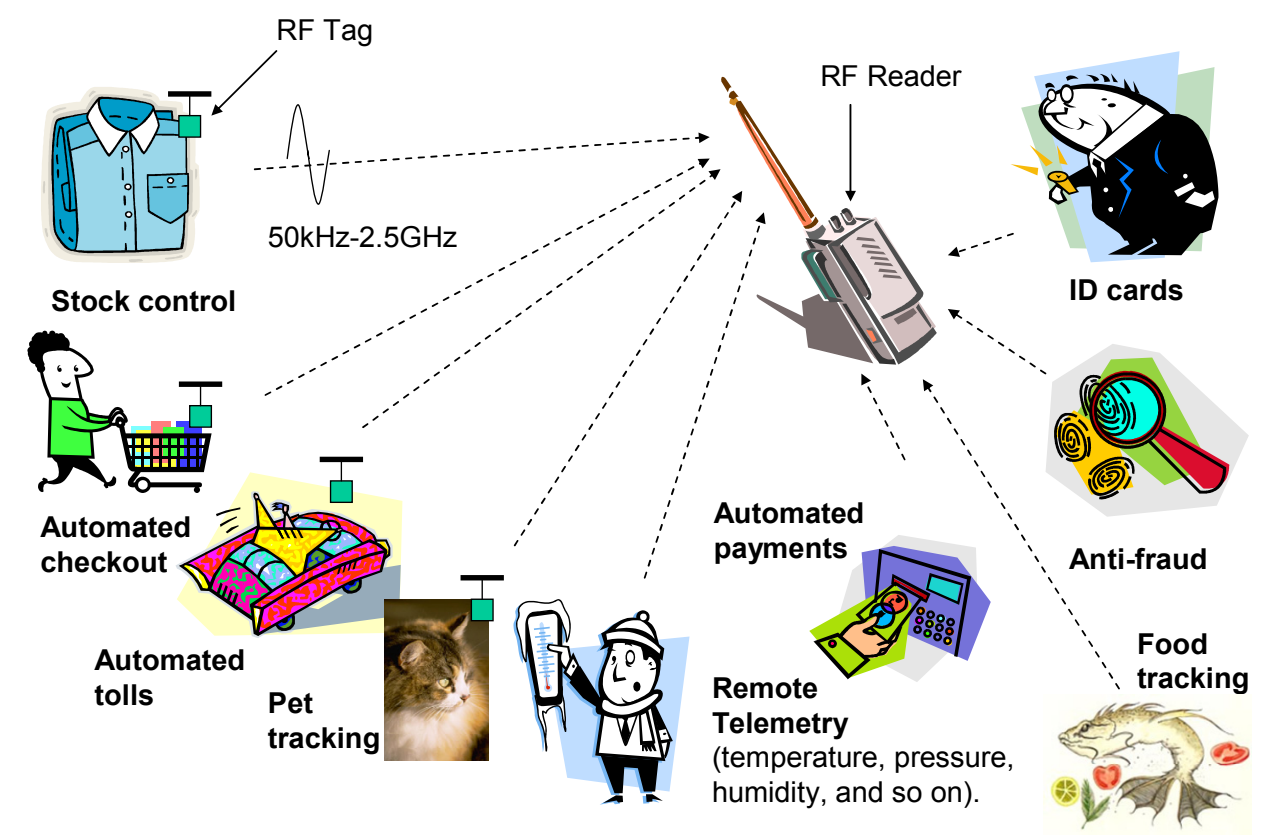

Figure 2: Applications of RFID

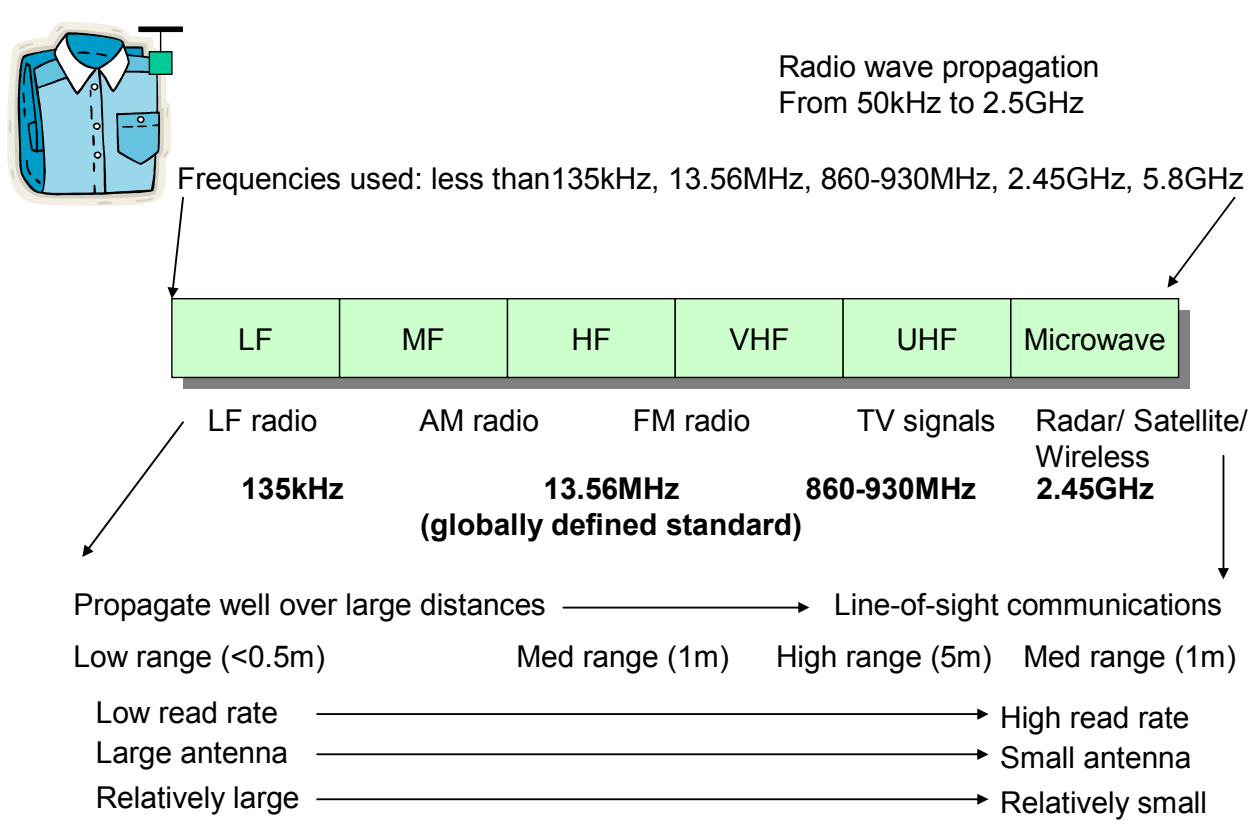

Figure 3: RFID radio spectrum

Along with the choice of operation frequency, a key design feature is whether the tag is active or passive. An active tag has its own power source, such as from a local battery, and is thus relatively large, but they have a good wireless range. A passive tag, on the other hand, do not have a local power source, thus they use the radio energy that is sent from an RF reader to couple enough energy to send back their identification. These types of tags are thus fairly small, but the lack the range of the active tags. Their great advantage, though, is that they are relatively inexpensive, when compared with active tags. The challenge in this research project is to use these tags to identify patients. An inexpensive method will thus allow new tags to be used for every patient in the hospital, and allow them to be destroyed when they leave, where active tags would be too expensive in costs if they were to be lost.

\section{LITERATURE REVIEW}

There has been a wide application of RFID, including the work done on sociophysiology in honeybees and its role for bee health [21]. With this research, a large number of bees were tagged with passive RFID tags, and was used to study their behaviour. The main focus for many RFID-based patient ID systems is on active tags which allow patients or machinery to be identified and located anywhere within designated 
areas $[7,8]$. The disadvantage of these tags, as previously said, is that they are relatively expensive, although it has been successfully introduced to high-risk areas, such as neo-natal wards where child abduction has become a serious issue in recent years $[9,10]$. Another application area is in surgery, where relatively simple setups have been used to write and read relatively small amount of information to/from passive RFID tags, in order to eradicate medical errors and to prevent litigation $[13,14]$.

There has been a growing interest in passive tagging recently [11]. Passive tags typically also do not actually store extensive patient information that needs to be protected. Also a passive tag does not need to be cleaned and disinfected as it is disposable, and they will not be re-used. Furthermore, they are not prone to over the air spoofing attacks and other manipulations with criminal intend [12].

Clinical trials with 1000 patients or so have been conducted in Germany and the US but in neither trial, the passive tags were directly linked to the central hospital patient data base $[15,16]$.

A key feature of an electronic system for the health sector is that it should comply with HIPAA statute that requires reasonable and appropriate safeguards to protect health information. Its main goals are [18]:

- Ensure the confidentiality, integrity, and availability of all electronic protected health information the covered entity creates, receives, maintains, or transmits.

- Protect against any reasonably anticipated threats or hazards to the security or integrity of such information.

- Protect against any reasonably anticipated uses or disclosures of such information that are not permitted or required under [the security rules].

- Ensure compliance with the [security rules] by its workforce.

Key objectives of the plan includes review and consideration of the following security-related areas:

- Design network communication configurations (wireless LAN, WAN, Internet) for strong encryption and audit trail reporting.

- Design organizational procedures regarding access to information.

- Develop transition plans for transmission of data not currently implemented into the LAN/WAN environment

- Consider recommended infrastructure needs such as new or additional computing devices (including wireless devices, laptops, Pocket PCs, and tablet PCs).

- Develop necessary training assistance for facility staff [19].

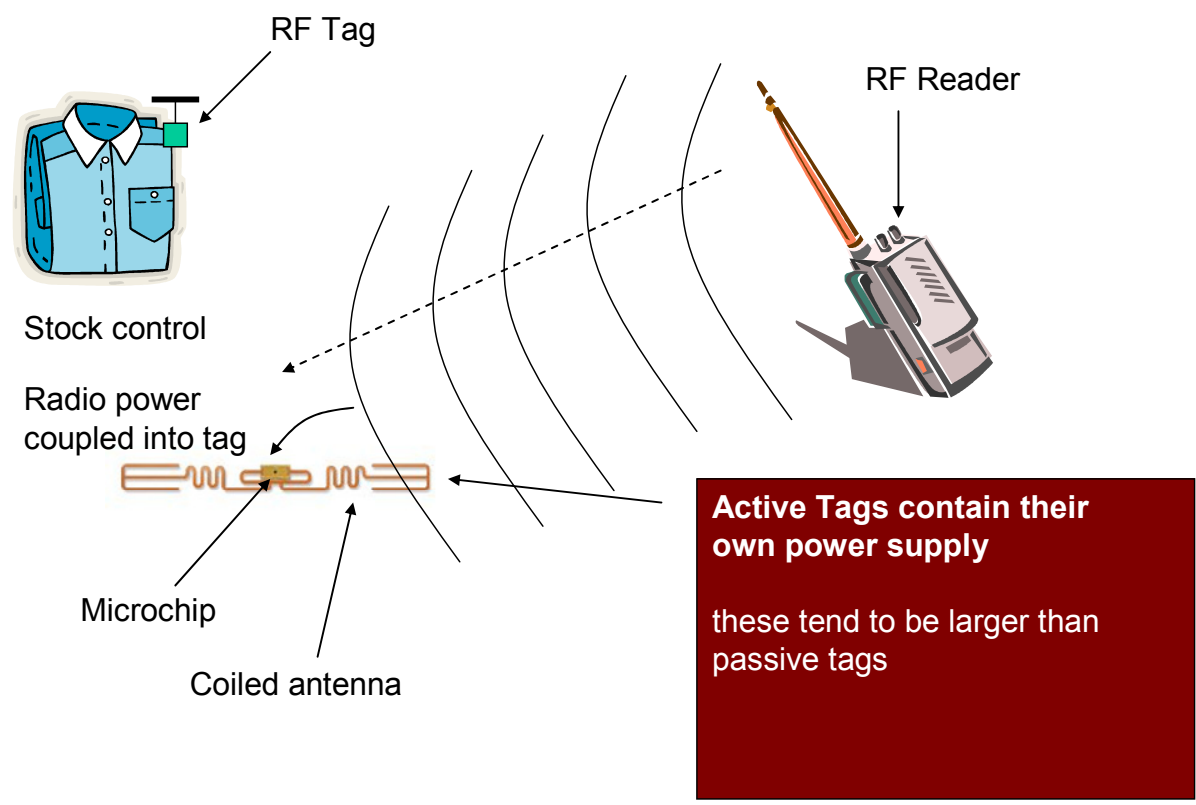

Figure 4: Passive tags

\section{V.DESIGN OBJECTIVES}

This design describes the implement framework which proposes to be a low cost system for hospitals, which is protected under UK patent law [17]. The design of the RFID system takes on several key features:

- Integration with the existing NHS database, which will be a read-only data transfer.

- Complete compatibility for all software elements for the software.
- Overcomes firewall problems, which can often block applications. Most NHS IT systems have extensive firewalls which do not allow the transfer of communication which do not conform to strict specifications.

- Soft linking of the RFID tag to the database.

- Coding of highly relevant conditions such as allergies or infectious disease by different colours of the display and standardisation of these colours.

- Logging of accesses to the patient. 


\section{FRAMEWORK IMPLEMENTATION}

Often software fails in its implementation, as elements of it do not fit together well, thus the proposed system using the .NET environment, which is a completely integrated software system that binds Windows programs with Web services, and will the underlying Windows networked infrastructure. Users and their roles can be easily authenticated through a Windows domain system. Along with this a major problem is often that firewalls block communications for unwanted TCP ports, this to overcome this problem the system uses Web Services, which, because they use the same TCP port as normal Web traffic, are not blocked by firewalls (Figure 5).

The local database thus stores the mapping of the RFID tag to the patient ID, and also the users on the system, which are authenticated again the main user account system. To simply the security of the system a range of roles have been defined, which defines the data that the viewer is allowed to view. These are:
- WARDCLerk. This role defines a registration role of the system, and the main details for the patient can be viewed, such as for their name, address, and so on, but no actual details can be shown.

- NuRSE. This defines a limited subset of the data that can be viewed, such as for the details of patient visits and notes. This role is setup from an administration panel.

- Consultant. This defines the highest level of viewing, and it is possible, through levels of authentication, to view the full details of the patient.

All of the accesses to the local database are achieved through Web services, thus no direct access can occur to the local database. Along with this, all of the data transferred between the clients and the Web service are encrypted before transmission and then decrypted by the Web service, and viceversa.

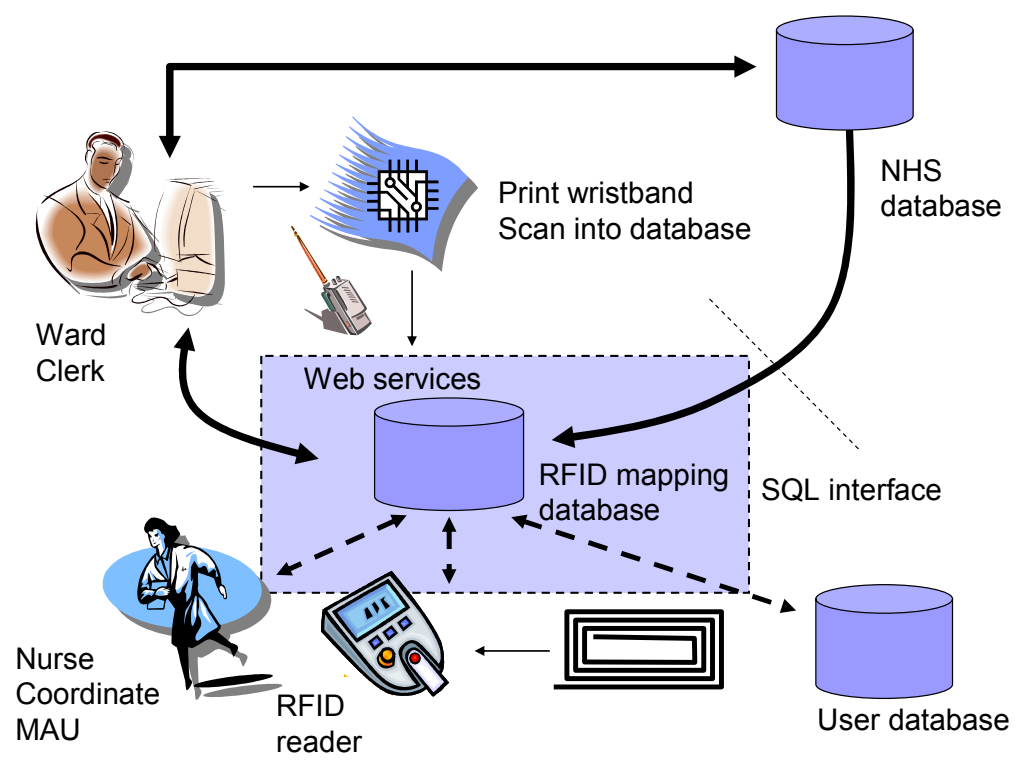

Figure 5: Framework with databases

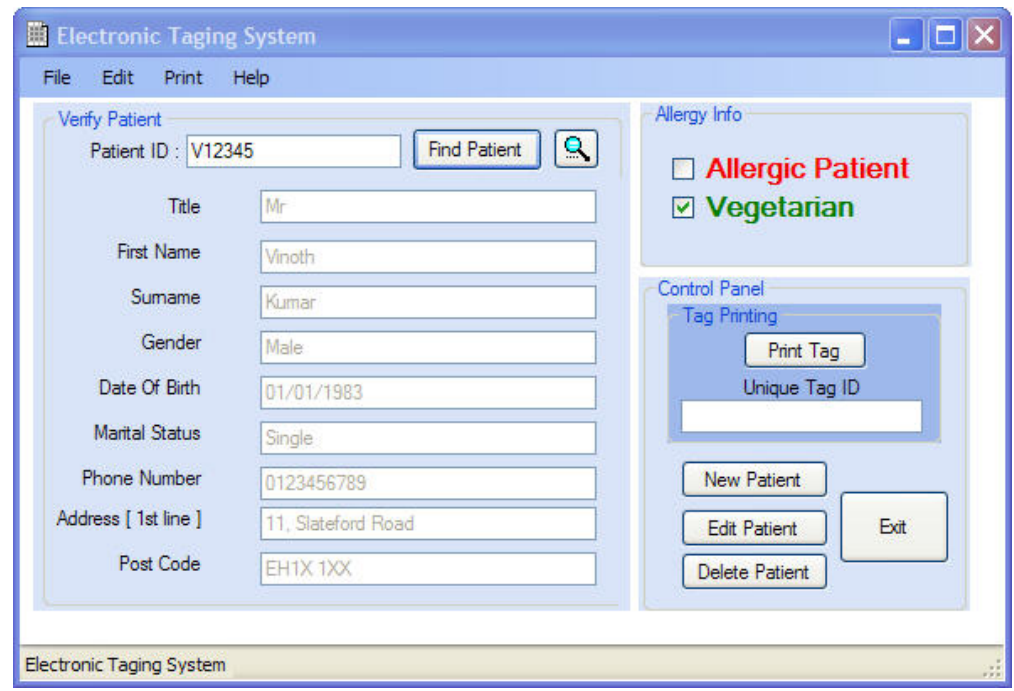

Figure 6: Initial registration 


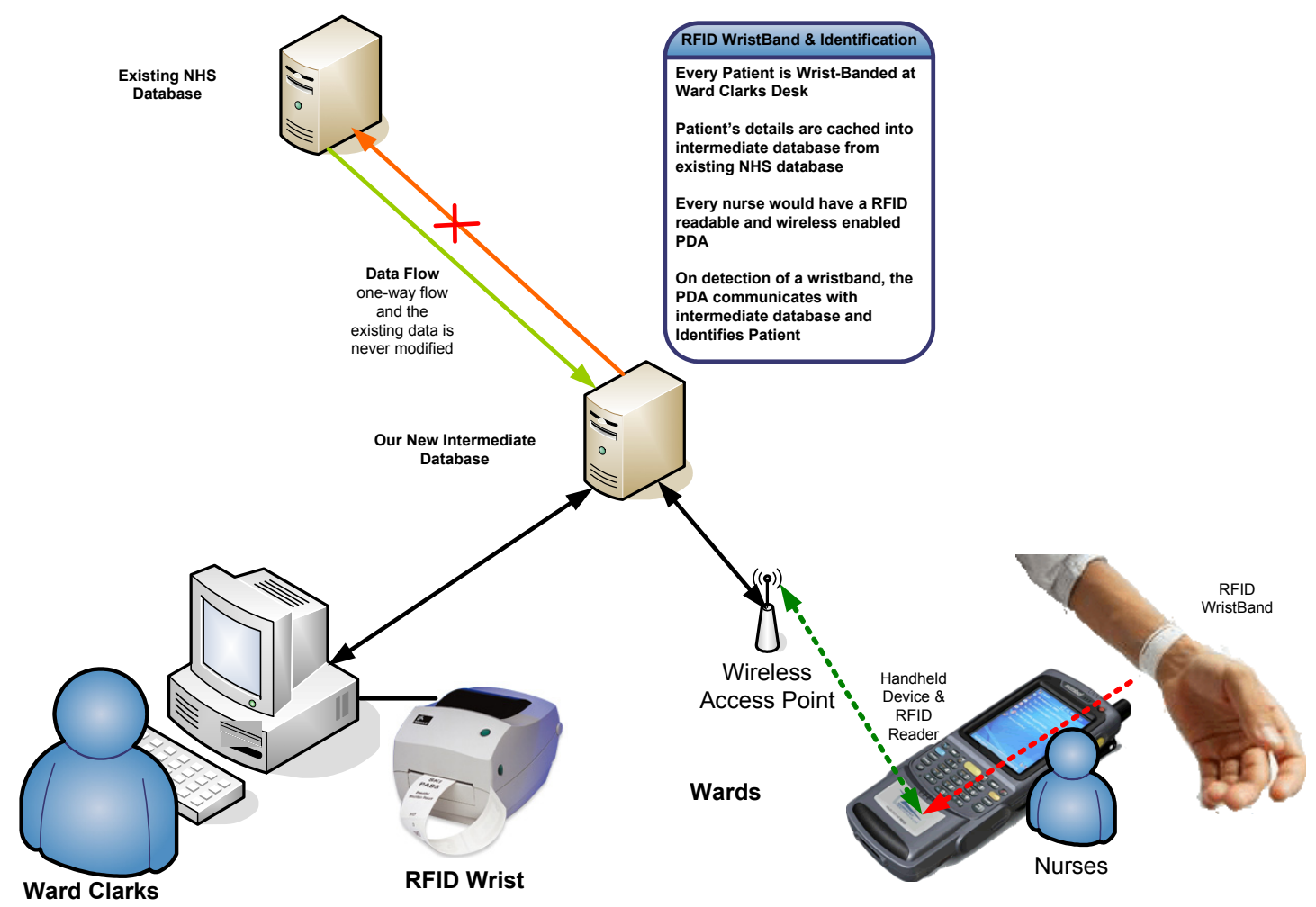

Figure 7: Overview of system

Figure 7 provides an overview of the system, where an RFID reader is used to read the wristband. The operation of the system is then:

\section{Initial registration}

Initially the Ward Clerk registers the patient, but accessing their Patient ID, and from this the details of the patient are downloaded into the local database. The Ward Clerk then print the RFID tag to a Zebra RFID printer. A key feature of the system is the color coding on any access for allergies and for vegetarian requirements. These have been color coded as red and green, respectively (Figure 8). As the tag is printed the RFID ID is then mapped in the database between the Patient ID and the tag. It is only this mapping which links the patient details to the tag (Figure 9).

\section{Usage}

The main details then displaying include name, DOB, sex, Hospital ID number, date and time of admission, current ward, link to previous admissions within the last 18months, link to note pads (nurses), link to note pad (doctors only), link to picture. Allergies are coded in red writing on the screen, such as for Penicillin, and yellow for infective, such as for MRSA, HIV, and so on.

A key factor in the adoption of the system is to create user interfaces which are not overloaded with information, but which should give links to other related data. An example of the Nurse screen for the PDA is shown in Figure 10 and for the consultant details in Figure 11. The security of the system is enhanced with the use of a role-based approach to the login procedure, where users login with the user role such as shown in Figure 12 and Figure 13.

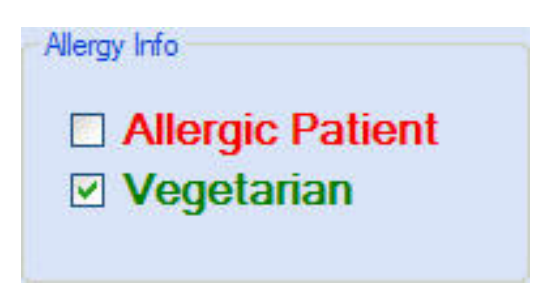

Figure 8: Allergy/vegetarian alert
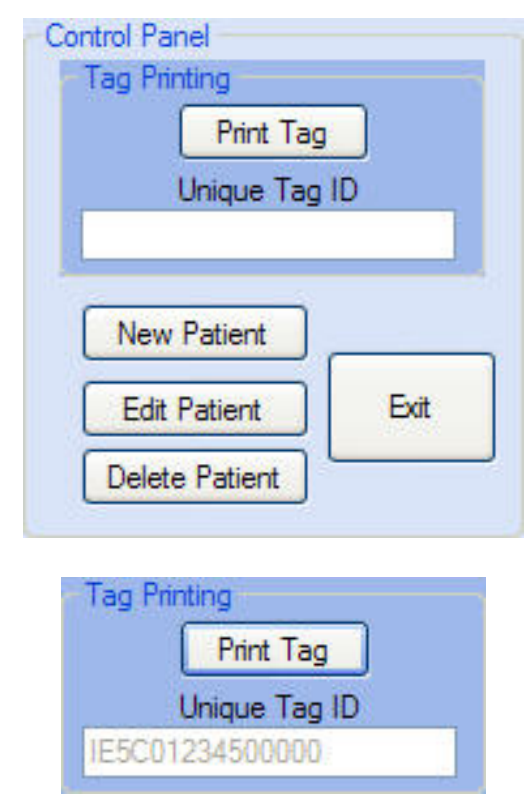

Figure 9: RFID tag details 
RFID Identifier G P.ll đর́ 4:35

\section{Tag Found}

\begin{tabular}{|c|c|c|}
\hline \multicolumn{3}{|c|}{$\begin{array}{l}\text { Nurse Mode } \\
\text { Logged in as: Vinoth }\end{array}$} \\
\hline \multirow{5}{*}{$\begin{array}{l}\text { Title } \\
\text { First Name } \\
\text { Surname } \\
\text { Gender } \\
\text { Date of Birth }\end{array}$} & \multicolumn{2}{|l|}{ Mr } \\
\hline & \multicolumn{2}{|c|}{ Vinoth } \\
\hline & \multicolumn{2}{|c|}{ Kumar } \\
\hline & \multicolumn{2}{|l|}{ Male } \\
\hline & \multicolumn{2}{|c|}{$01 / 01 / 1983$} \\
\hline \multicolumn{3}{|c|}{ Click here for more... } \\
\hline \multicolumn{2}{|c|}{ FindConsultant } & Daily Logs \\
\hline Start & Retry & Exit \\
\hline
\end{tabular}

Figure 10: Main PDA screen interface (Nurse)

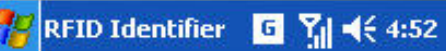

\section{Tag Found}

\begin{tabular}{|c|c|c|}
\hline \multicolumn{3}{|c|}{$\begin{array}{l}\text { Consultant Mode } \\
\text { Logged in as : Vinoth }\end{array}$} \\
\hline \multirow{6}{*}{$\begin{array}{l}\text { Title } \\
\text { First Name } \\
\text { Surname } \\
\text { Gender } \\
\text { Date of Birth } \\
\text { Phone No }\end{array}$} & \multicolumn{2}{|l|}{ Mr } \\
\hline & \multicolumn{2}{|c|}{ Vinoth } \\
\hline & \multicolumn{2}{|c|}{ Kumar } \\
\hline & \multicolumn{2}{|l|}{ Male } \\
\hline & \multicolumn{2}{|c|}{$01 / 01 / 1983$} \\
\hline & \multicolumn{2}{|c|}{0123456789} \\
\hline \multicolumn{3}{|c|}{ Click here for more... } \\
\hline Consultant & GP & Daily Logs \\
\hline Start & Retry & Exit \\
\hline
\end{tabular}

Figure 11: Main PDA screen interface (Consultant)

RFID Identifire - G ?

\section{RFID WristBand Wand}

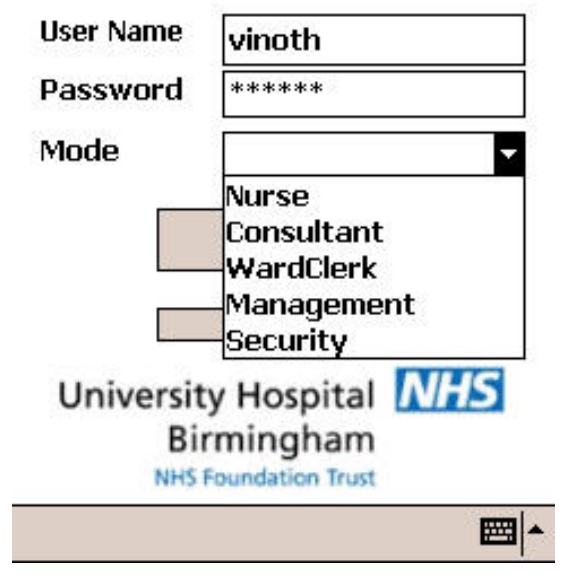

Figure 12: Role based security for login
RFID Identifire - G ?.ll $4<$ 4:08

\section{RFID WristBand Wand}

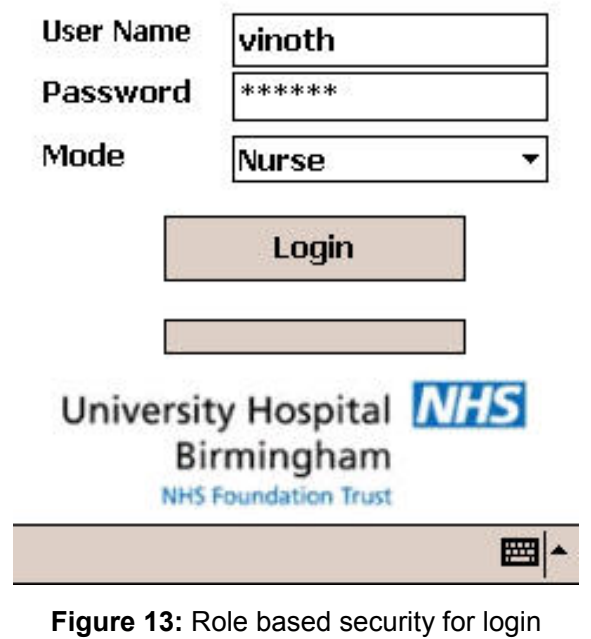

\section{CONCLUSIONS}

Our approach not only allows unique patient identification but in addition offers intelligent features which could be used to protect vulnerable adults within the hospital environment. Patients fitted with RFID wristbands will be clearly identifiable and it will prevent those high-risk patients to leave the hospital or certain wards accidentally or enter potentially dangerous areas like $\mathrm{X}$-ray departments.

\section{VIII.REFERENCES}

[1] National Blood Service and Royal College of Physicians. Comparative Report for Blood Transfusion in England. November 2003.

[2] Guthrie, D. Positive Patient Identification. Frontiers in Laboratory Medicine. The Royal College of Physicians: London, February 2003.

[3] Human Reliability Associates. Mismatching between planned and actual treatments in medicine - manual checking approaches to prevention. 2004. Available at www.npsa.nhs.uk

[4] Cambridge Consultants. Ensuring patients are correctly matched with samples or specimens taken from them and treatment planned for them. January 2004. Available at www.npsa.nhs.uk

[5] Royal College of Nursing. Right Blood, Right Patient, Right Time, RCN guidance for improving transfusion practice. London.

[6] http://www.npsa.nhs.uk/site/media/documents/ 1440_Safer_Patient_Identification_SPN.pdf

[7] Fry EA, Lenert LA, MASCAL: RFID tracking of patients, staff and equipment to enhance hospital response to mass casualty events. AMIA Ann Symp Proceedings, 2005; 261-5

[8] Davis S, Tagging along. RFID helps hospitals track assets and people. Health Facil Manage 2004 Dec;17(12):20-4.

[9] http://www.verichipcorp.com/content/solutions/hugs 
[10] http://www.verichipcorp.com/content/solutions/halo

[11] Malkary G, Active RFID popular pick. ROI for the passive form is weak, but overall prospects look better, says study. Healthc Inform 2006 Feb;23(2):108

[12] Halamka J, Jules A, Stubblefield A, Westhues J, The Security Implications of VeriChip Cloning. J Am Med Inform Assoc, 2006 Aug [Epub ahead of print]

[13] Macario A, Morris D, Morris S, Initial clinical evaluation of a handheld device for detecting retained surgical gauze sponges using radiofrequency identification technology. Arch Surg. 2006 Jul;141(7):659-62

[14] http://www.bjhc.co.uk/news/1/2005/n502016.htm
[15] http://www.rfidjournal.com/article/articleprint/2169/$1 / 1$

[16] Collins J, RFID Delivers Healthy Return for Hospitals. RFID Journal, May 2nd 2005.

[17] Patent application number GB0619694.3.

[18] http://wireless.ittoolbox.com/pub/MM020102.pdf

[19] http://www.hipaadvisory.com/tech/wireless.html. Proposed Hippa Solution

[20] http://www.npsa.nhs.uk/press/display? contentId=4405

[21] http://www.biozentrum.uni-wuerzburg.de/fileadmin /REPORT/ZOO2/zoo2006.htm 